Collection SFN 7 (2007) 221-240

(C) EDP Sciences, Les Ulis

DOI: $10.1051 / \mathrm{sfn}: 2007024$

\title{
Techniques de diffusion des neutrons en incidence rasante pour les nanosciences
}

\author{
V. Lauter-Pasyuk ${ }^{1,2}$ \\ ${ }^{1}$ Physik Department, TU München, 85747 Garching, Germany \\ ${ }^{2}$ Institut Laue-Langevin, 6 rue Jules Horowitz, 38042 Grenoble, France
}

\section{INTRODUCTION}

La diffusion des neutrons en incidence rasante et l'étude des proprietés de structures dites de "dimensionnalité réduite" connaissent une activité croissante dans le cadre de la recherche en matière condensée.

Les développements des techniques d'élaboration de films minces ont crée un besoin important en matière de caractérisation des proprietés de ces films minces. Parmi ces nombreuses techniques de caractérisation, les techniques neutroniques offrent des avantages précieux que l'on peut résumer en quelques lignes :

- la variation de contraste entre différent élements ou isotopes liée aux sections efficaces de diffusion des noyaux atomiques et la nature nucléaire de l'interaction rendant cette technique peu sensible aux nuages électroniques à l'inverse des rayons-X,

- la très grande profondeur de pénétration des neutrons et l'accès aux surfaces enterrées en raison de la faible absorption,

- un accès direct à la distribution d'aimantation dans les couches minces ou les multicouches, ceci grâce à l'interaction dipolaire entre le neutron et le champ électromagnétique crée par les moments magnétiques du matériau,

- la diffusion des neutrons est une technique non destructive même pour les systèmes biologiques.

Depuis le début des années 80 la réflectivité spéculaire des neutrons s'est affirmée comme une technique expérimentale éprouvée pour l'étude des films minces et des multicouches. Aujourd'hui les réflectomètres sont utilisés pour étudier aussi bien les systèmes magnétiques, les matériaux organiques et biologiques que les films de polymères (voir revue [1]). A l'heure actuelle seule la réflectométrie spéculaire est couramment utilisée pour étudier la structure des films ou des multicouches perpendiculairement à leurs surfaces. La réflexion spéculaire permet d'accéder aux informations sur le profil vertical de la densité moyenne de longueur de diffusion (scattering lenght density ou SLD) du matériau, moyennée sur l'ensemble de la surface. Cependant, en réalité, la réflexion spéculaire pure n'existe pas car les surfaces ou interfaces ne sont pas idéales, ne sont pas parfaites à l'échelle atomique. En conséquence, la réflexion spéculaire s'accompagne toujours d'une contribution hors-spéculaire.

L'ensemble de l'intensité hors spéculaire diffusée n'est accessible qu'avec l'aide de détecteurs bidimensionnels. La diffusion hors spéculaire rend compte des structures latérales (facteur de forme latéral, facteur de structure, rugosité de surface ou d'interface) des surfaces et interfaces des films ou multicouches.

Une information complète de la structure 3D (transverse et latérale) des multicouches peut donc être obtenue en utilisant une combinaison de diffusion en incidence rasante des neutrons (tels aussi les rayons $\mathrm{X}$ ) en considérant les diffusions spéculaires et hors spéculaires.

Les progrès en fabrication et caractérisation de surface ont rendu possible la production de nouveaux types de matériaux structurés à l'échelle nanométrique. Quelques uns des meilleurs exemples de ces matériaux sont les multicouches magnétiques développées en vue de produire des tâtes de lecture pour les dispositifs de stockage magnétique de données. Ils consistent en structures "sandwich" de 
couches magnétiques séparées par un espaceur non magnétique. La résistance électrique de l'ensemble change sous l'effet du champ magnétique généré par le grain magnétique formant le "bit" d'information. Cet effet est appelé "magnéto-résistance géante" ou GMR [2,3]. Ces structures sont appelées "vannes de spins" car elles transmettent préferentiellement les électrons ayant une valeur de spin bien définie [4,5]. Un autre phénomène, analogue au précédent, est l'oscillation de l'orientation magnétique de deux couches en fonction de l'épaisseur de l'espaceur non-magnétique. Les techniques comme la réflectivité des neutrons polarisés en condition hors spéculaire permettent donc d'accéder aux détails des structures des matériaux GMR. Le cas expérimental concret du multicouche $\mathrm{Fe} / \mathrm{Cr}(001)$, à effet GMR, est représenté sur la Figure 1(a) [33].

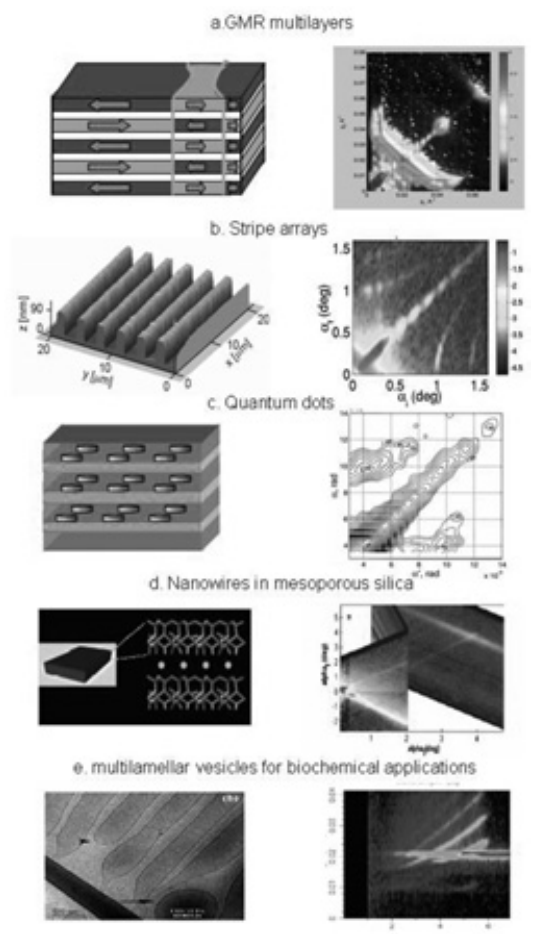

Figure 1. Sur la gauche de la figure, nanostructures dans l'espace réel. Sur la droite, les mêmes nano-structures vues par les neutrons lors d'expériences en incidence rasante. (a) Multicouches $\mathrm{Fe} / \mathrm{Cr}$ (ML), constituées de couches magnétiques (rouges et roses), présentant une forte anisotropie planaire et des domaines d'aimantation alternée (indiqués par les flèches bleues) dus au couplage d'échange via les espaceurs non magnétiques (en blanc); carte à 2 dimensions de l'intensité réfléchie et diffusée par cette multicouche. (b) Réseau de 'stripes' magnétique périodiques ayant un axe facile le long des 'stripes' ; la carte 2-D de l'intensité est déterminée par la structure périodique latérale, les domaines d'ondulation et les corrélations dans l'ensemble. (c) Réseau de plots de Fer ; l'intensité diffusée dépend de la forme, de la taille, de la distribution et de l'aimantation des plots. (d) Film composite de nanofils métalliques insérés dans une matrice mésoporeuses de silice; l'intensité 2D mesurée donne des informations sur la taille des nanofils, la dispersion de diamètres, la distribution et l'orientation à l'intérieur de la matrice. (e) Spherulites, ou vésicules multilamélaires qui se forment spontanément dans les surfactants cationiques à faible concentration. De l'intensité réfléchie et hors-spéculaire, on peut extraire le comportement complexe, les transitions de phases la taille des vésicules multilamélaires ainsi que les détails de leur structure transverse et latérale.

Récemment la réflectivité des neutrons polarisés a été utilisée sur $\mathrm{VCo}_{0.7} \mathrm{Fe}_{0.3}$, un réseau de bandes ("stripes") magnétiques préparé par lithographie à jets d'électrons (electron beam lithography) et par 
des techniques de gravure ("etching") [6]. Comprendre l'interaction magnétique entre bandes voisines est important pour les applications en magnéto-électronique. Le renversement d'aimantation du réseau de bandes, l'anisotropie uniaxiale induite, la structure en domaines et les corrélations entre bandes ont été obtenues en utilisant les résultats de réflectivité des neutrons (voir exemple sur la Figure 1(b)).

De nos jours il devient possible de produire des réseaux périodiques de plots sous-microniques sur des surfaces très plates ou à l'intérieur de films ou multicouches semiconducteurs, métalliques ou supraconducteurs qui sont au coeur des applications liées au stockage optique ou magnétique de données [7-9].

Une des premières expériences sur un réseau de plots de Fe (diamètre $300 \mathrm{~nm}$, hauteur $80 \mathrm{~nm}$ ) a été réalisée avec des neutrons polarisés le long de l'aimantation des plots (Figure 1(c)). L'aimantation des plots, leurs formes, tailles et distribution périodique moyennée sur l'ensemble de l'échantillon a ainsi pu être obtenue [10].

Les nanofils magnétiques insérés dans les matrices mésoporeuses de silice, étant des matériaux très ordonnés, sont également des nanostructures importantes pour les dispositifs de stockage. Les premières expériences de diffusion des neutrons ont été réalisées pour évaluer l'influence des conditions de préparation sur les proprietés magnétiques et structurales de ces nanofils [11] (Figure 1(d)). A partir des expériences de neutrons on peut obtenir des informations sur l'influence des paramètres d'anisotropie du métal magnétique liés aux cavités créées par ces nanoréacteurs que sont les structures nanoporeuses (zéolites, silice mésoporeuse, membranes d'alumine).

Pour la création de biosenseurs basés sur des membranes, le développement d'interfaces biocompatibles joue un rôle important [12]. De petites vésicules phospolipidiques unilamellaires sont les ingrédients de base pour obtenir des systèmes biophysiques modèles permettant l'étude des interactions de ces biomolécules avec la surface des membranes [13]. A ce titre, la réflectivité des neutrons a été utilisée pour étudier in situ la formation de bicouches phospolipidiques [14]. Les vésicules lamellaires sont particulièrement intéressantes en biochimie et en pharmacie pour l'administration de médicaments $[15,16]$. Les avancées récentes dans les systèmes médicamenteux ont mis l'accent sur des systèmes surfactants alternatifs qui peuvent produire des structures similaires [17].

Les systèmes contenant des mélanges de surfactant cationique et anionique ou des surfactants nonioniques à une seule chaîne peuvent ainsi former des solutions vésiculaires. La structure lamellaire ordonnée et la stabilité des surfactants formés de bi-chaînes cationiques ont été étudiées par réflectivité des neutrons [18], et la structure interfaciale des agrégats a été examinée par diffusion hors spéculaire (Figure 1 (e)).

Bien que la liste des expériences soit encore longue, on peut déjà se rendre compte à partir de ces brefs rappels que les techniques de diffusion des neutrons sur les surfaces et les structures nanométriques sont de plus en plus couramment utilisées dans tous les domaines intéressant les nanosciences. la raison profonde est que la production d'objets de taille nanométrique demande un contrôle parfait des structures, taille, formes et symétries des objets. L'utilisation de la réflectivité des neutrons permet d'accéder à ces informations sur l'ensemble du volume du matériau, en opposition avec les sondes locales comme la microscopie à transmission d'électrons (TEM) et, élement non negligeable, sans dégrader les matériaux.

\section{LA LONGUEUR DE COHÉRENCE DES NEUTRONS}

Les informations sur la structure 3D (transverse et latérale) sont encodées dans l'intensité des neutrons réfléchie et diffusée par l'échantillon. Les exemples de la Figure 1 illustrent bien la variété des cartographies 2D de l'intensité diffusée reflétant ainsi la complexité des objets étudiés. La forte intensité observée le long de la diagonale (Figure 1(a)-(d)) ou horizontalement (Figure 1(e) correspond à la réflexion spéculaire. Elle est déterminée par le profil vertical (normal à la surface) de la densité moyenne de longueur de diffusion (SLD pour Scattering Lenght Density) qui correspondà la structure transverse du système moyennée sur l'ensemble de la surface de l'échantillon. Les déviations autour de cette valeur 
moyenne (fluctuations latérales de la SLD) donnent lieu à une diffusion hors spéculaire autour de la réflection spéculaire.

\section{Quand observer de la diffusion hors spéculaire?}

Cela arrive quand la taille latérale des fluctuations est plus petite que la projection latérale de la longueur de cohérence des neutrons. La taille latérale des fluctuations est souvent décrite par la longueur de corrélation qui est la distance entre deux points qui diffusent en phase [19]. Pour estimer la longueur de cohérence des neutrons dans l'échantillon, nous considérons la géometrie de diffusion de la réflectivité. Les sources de neutrons produisent une radiation non-cohérente, donc nous ne discuterons pas de la longueur de cohérence de la source. La longueur de cohérence des neutrons est déterminée uniquement par la cohérence (ou résolution) temporelle (longueur d'onde) ou spatiale (angulaire) du faisceau. Une source de neutrons ponctuelle parfaitement monochromatique n'existe pas, et par conséquence la pureté spectrale (largeur spectrale) $\delta \lambda / \lambda$ de la source détermine la cohérence temporelle (cohérence longitudinale), alors que la résolution angulaire $\delta \alpha$ (ou la taille) de la source détermine la cohérence latérale (transverse).

\subsection{Largeur de bande spectrale et longueur de cohérence longitudinale des neutrons}

La longueur de cohérence longitudinale des neutrons est définie comme la distance sur laquelle une radiation de largeur spectrale $\delta \lambda$ devient déphasée de $\pi$, comme le montre la Figure 2. Pour une longueur d'onde $\lambda$ se propageant sur $n$ périodes, la longueur de cohérence longitudinale est :

$$
L_{l}=\lambda n
$$

Pour une longueur d'onde $\lambda+\delta \lambda$

$$
L_{l}=(\lambda+\delta \lambda)\left(n-\frac{1}{2}\right)
$$

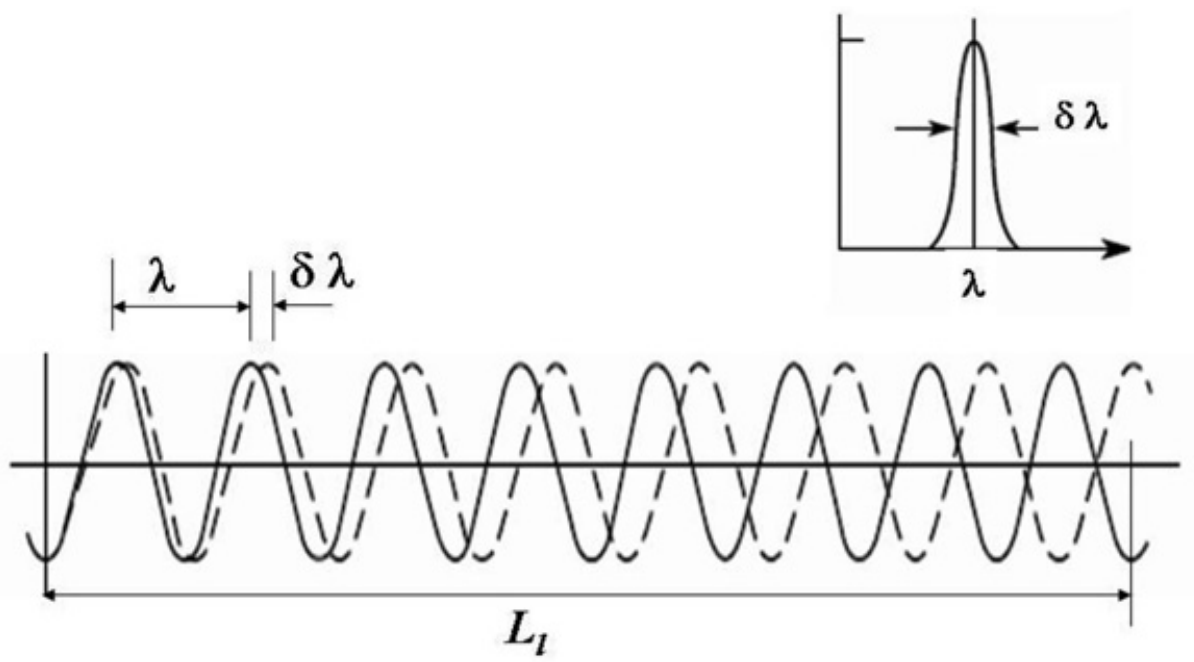

Figure 2. Longueur de cohérence longitudinale (temporelle). C'est la distance parcourue par les neutrons pendant le temps de cohérence, qui détermine la profondeur maximum de l'objet étudié dans une expérience de réflectivité. 
D'après (2.1) et (2.2), on peut facilement obtenir l'expression de la longueur de cohérence longitudinale sous une autre forme

$$
L_{l}=\frac{\lambda^{2}}{2 \delta \lambda}
$$

La longueur de cohérence longitudinale définit ainsi la plus grande différence de chemin pour laquelle une interférence est possible. Par ailleurs, on peut aisément dériver que, dans une expérience de réflectivité, la différence de chemin entre les ondes réfléchies par la surface et par le substrat est en général plus petite que $L_{l}$.

\subsection{Résolution angulaire et longueur de cohérence latérale des neutrons}

En vertu du principe d'incertitude de Heisenberg, le quantité de mouvement du neutron devient de moins en moins définie à mesure que la position du faisceau devient, elle, plus définie.

Une source parfaitement ponctuelle n'existant pas, la résolution angulaire a une valeur finie. Celle-ci détermine la longueur de cohérence latérale des neutrons.

A partir de chaque point de l'échantillon, on voit une source (ou un collimateur qui détermine la résolution) de taille $a$ à une distance $R$ avec un angle de divergence $\phi_{i}$. En général $R>>a$. A partir de ces considérations géométriques (Figure 3), la longueur de cohérence latérale $L_{s}$ (la longueur de cohérence dans la section du faisceau incident) est donnée par

$$
L_{s}=\lambda \frac{D}{2 a}=\frac{\lambda}{2 \phi}=\frac{\lambda}{\delta \alpha}
$$

où $\delta \alpha$ est la résolution angulaire.

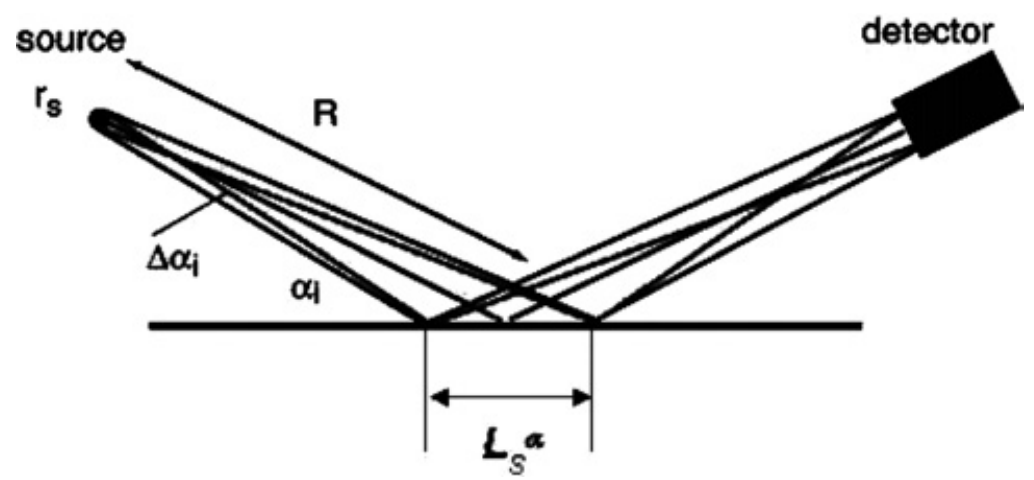

Figure 3. Longueur de cohérence spatiale $L_{s}^{\alpha}$ des neutrons à la position de l'échantillon. $R$ est la distance à la source (de dimension linéaire $r_{s}$ ), $\alpha$ est l'angle d'incidence, $\Delta \alpha$ est la divergence angulaire.

Pour les faibles angles d'incidence $\alpha$, il faut tenir compte des projections sur la surface de l'échantillon des deux longueurs de cohérence à la fois. Il vient ainsi :

$$
\begin{aligned}
& L_{l}^{\alpha}=\frac{L_{l}}{\cos \alpha}=\frac{\lambda^{2}}{2 \alpha \delta \lambda} \\
& L_{s}^{\alpha}=\frac{L_{s}}{\sin \alpha}=\frac{L_{s}}{\alpha}=\frac{\lambda}{\alpha \delta \alpha}
\end{aligned}
$$

Au cours d'une expérience de réflectivité, la projection de la longueur de cohérence latérale n'est pas une constante et peut même changer d'un facteur 10-15. Dans le cas d'un faisceau monochromatique, 
elle change en fonction de l'angle d'incidence qui est la variable expérimentale principale. Dans le cas d'une expérience en temps-de-vol à angle d'incidence fixe, $L_{s}^{\alpha}$ est une fonction de la longueur d'onde incidente.

Les mêmes considérations s'appliquent pour la longueur de cohérence déterminée par la résolution spatiale des détecteurs et par la distance entre l'échantillon et les détecteurs. Ainsi, pour la détermination de la longueur de cohérence globale il faut tenir compte des neutrons incidents aussi bien que des neutrons diffusés.

A partir de considérations simples, on trouve que $L_{s}^{\alpha}$ peut être de l'ordre de plusieurs centaines de microns le long du faisceau. Ainsi, la longueur de cohérence latérale dans le plan du film est grande par rapport aux longueurs latérales caractéristiques des nano-objets (plots, domaines, inhomogénéites, etc.) de surface mais petite par rapport aux dimensions physiques de l'échantillon (plusieurs millimètres en général). Donc l'intensité diffusée est la somme incohérente de la diffusion générée par des parties statistiquement indépendantes de l'échantillon.

\section{DIFFUSION EN INCIDENCE RASANTE ET APPROXIMATION DE BORN DE L'ONDE DISTORDUE}

Traditionellement, dans une expérience de diffraction, la diffusion des neutrons est calculée dans le cadre de l'approximation de Born combinée à un pseudopotentiel de Fermi paramétré par la longueur de diffusion atomique. Dans ce cadre, une onde plane diffusée par un diffuseur faible n'est pas trop perturbée par le processus de diffusion. Cette approximation donne de bons résultats lorsqu'elle s'applique à la diffraction ou à la diffusion aux petits angles car la plupart des neutrons ne sont pas déviés lors de la diffusion. En revanche, lors d'une expérience de réflectivité, spécialement près de la réflexion totale, seule une faible part des neutrons est transmise à travers l'échantillon et le plus grand nombre de ces neutrons est réfléchi.

Une manière plus appropriée d'effectuer un calcul perturbatif de la réflectivité est de trouver les fonctions d'ondes des neutrons en interaction avec le potentiel moyen de la surface moyenné sur la surface que l'on définit par la coordonnée $\rho$. Pour calculer la diffusion hors-spéculaire, il convient de procéder à un calcul perturbatif en utilisant les fonctions d'ondes ainsi obtenues et de considérer un potentiel perturbatif rendant compte des déviations au potentiel moyen. Cette théorie de perturbations est connue sous le nom d'approximation de Born de l'onde distordue (DWBA pour "distorted wave Born approximation") [20]. Cette théorie a été pour la première fois utilisée par Vineyard [21] dans le cas de la diffraction de surface en incidence rasante et, peu de temps après, à l'étude de la structure de l'interface liquide-vapeur d'un métal liquide [22]. Plus tard, Sinha et al. [23] et R. Pynn [24] ont utilisé l'approche DWBA pour calculer la réflectivité spéculaire et la diffusion diffuse de surfaces rugueuses. La technique fût developpée plus avant en particulier pour l'étude de la diffusion hors-spéculaire magnétique [25] et non-magnétique [26] en utilisant le formalisme de super-matrices [27].

\subsection{Réflectivité spéculaire}

Considérons une expérience de réflectivité comme schématisée sur la Figure 4. Les neutrons incidents de vecteur d'onde $\mathbf{k}_{\mathbf{i}}$ sont réfléchis spéculairement avec un vecteur d'ondes $\mathbf{k}_{\mathbf{f}}$. Dans ce cas l'angle d'incidence $\alpha_{i}$ est égalà l'angle de réflexion $\alpha_{f}$. Si, à une échelle plus petite que la projection de la longueur de cohérence $L_{s}^{\alpha}$, la variation du potentiel d'interaction est une fonction de la seule coordonnée z perpendiculaire à la surface de l'échantillon, $V_{0}(z)$, alors les interfaces peuvent être considérées comme "latéralement plates". Alors, la solution de l'équation de Schrödinger

$$
\left\{\nabla^{2}+k_{0}^{2}-\left(2 m / \hbar^{2}\right) V_{0}(z)\right\}|\Psi(\mathbf{r})\rangle=0
$$


au dessus de la surface peut être écrite comme

$$
|\Psi(\mathbf{r})\rangle=\exp (i \boldsymbol{\kappa} \boldsymbol{\rho})\left\{e^{i p_{i} z}+\widehat{R} e^{-i p_{i} z}\right\}|\psi(0)\rangle=0
$$

où $\mathbf{k}=\{\hat{\mathbf{e}}, p\}$ est le vecteur d'onde du neutron. $k=2 \pi / \lambda, \boldsymbol{\kappa}$ est sa composante latérale tandis que $p_{i}$ est sa composante normale à la surface. $|\Psi(\mathbf{r})\rangle$ est vecteur des états de spins, $\widehat{R}$ est la matrice de réflectance. A l'intérieur de l'échantillon le champ d'ondes est formé par les ondes transmises a travers l'échantillon mais aussi par celles réfléchies par chaque interface éventuelle. Les opérateurs de transmission $\hat{t}$ et de réflexion $\hat{r}$ sont obtenus à partir des conditions aux limites. Le vecteur des états de spins se transforme en :

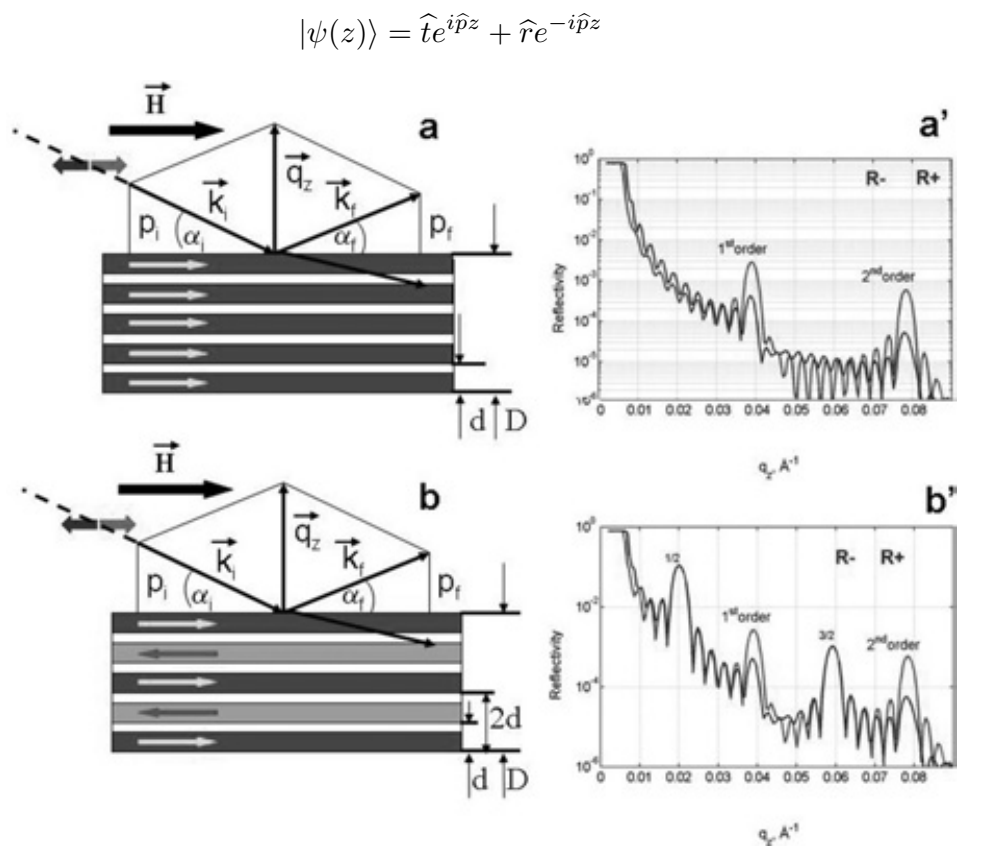

Figure 4. Schéma d'une expérience de réflectivité. (a) Multicouche magnétique (constituée d'un motif de deux couches d'épaisseur $d$ se répétant sur une épaisseur totale D), dans un champ magnétique saturant $\boldsymbol{H} ; \boldsymbol{k}_{i}$ et $\boldsymbol{k}_{f}$ sont les vecteurs d'onde des neutrons incidents sous un angle $\alpha_{i}$ et réfléchis de manière spéculaire sous l'angle $\alpha_{f}$. (a') Profils de réflectivité $R^{+}$et $R^{-}$en fonction du transfert de moment $q_{z}$; les positions des pics de Bragg sont déterminées par l'épaisseur du motif $\mathrm{d}$, alors que les franges de Kiessig [30] sont dues à l'épaisseur totale du film D. (b) Multicouche dans un champ magnétique extérieur rémanent résultant en un alignement opposé de l'aimantation dans deux couches adjacentes. (b') Les profils de réflectivité $R^{+}$et $R^{-}$présentent, en plus des pics de Bragg de la Figure 4(a), des pics $1 / 2$ et $3 / 2$ caractéristiques du doublement de la partie magnétique du profil de diffusion.

Dans le cas des matériaux magnétiques, le champ magnétique moyen est $\mathbf{B}=\mathbf{H}+4 \pi \mathbf{M}$, ou $\mathbf{H}$ est le champ magnétique externe et $\mathbf{M}$ est l'aimantation planaire moyenne. L'axe de quantification est le long de $\mathbf{B}$, les valeurs propres de l'opérateur $\hat{p}$ s'écrivent donc

$$
p^{ \pm}=\left(p_{i}^{2}-p_{N}^{2} \mp p_{M}^{2}\right)^{1 / 2}
$$

avec $p_{N}=\sqrt{4 \pi n b_{N}}$ et $p_{M}=\sqrt{2 m \boldsymbol{\mu B}}$, ou $n b_{N}$ est la densité de longueur de diffusion nucléaire. $\mu$ est le moment magnétique du neutron. A partir des conditions de continuité aux interfaces entre couches d'épaisseur $d_{n}$ des fonctions d'ondes et de leurs premières dérivées on en déduit les équations :

$$
\widehat{t}_{n} e^{i p_{n} d_{n}}+\widehat{r}_{n} e^{-i p_{n} d_{n}}=\widehat{t}_{n+1}+\widehat{r}_{n+1}
$$




$$
\left(\widehat{t}_{n} e^{i p_{n} d_{n}}-\widehat{r}_{n} e^{-i p_{n} d_{n}}\right) \widehat{p}_{n}=\left(\widehat{t}_{n+1}-\widehat{r}_{n+1}\right) \widehat{p}_{n+1}
$$

Une solution de ces équations peut être trouvée soit par le formalisme de super-matrices [27] soit par une méthode itérative exacte utilisant la relation de récursion de Parrat [28].

Un exemple de calculs de courbes de réflectivité d'une multicouche magnétique possédant des interfaces parfaites est représentée sur la Figure 4. Il s'agit d'un système "typique" de structure en multicouches GMR, composé de 12 couches identiques de Fe avec une anisotropie uniaxiale dans le plan séparées par des couches non-magnétiques de $\mathrm{Cr}$ sur un substrat de Si. La direction du champ magnétique externe est choisie le long de l'axe facile d'aimantation. A la saturation (Figures 4(a), 4(a')) les couches sont complètement aimantées le long du champ externe. Dans ce cas, seules les courbes de réflectivité "non-spin flip" (NSF), $R^{++}$and $R^{--}$, sont non nulles. Elles correspondent aux deux orientations de la polarisation des neutrons. Les courbes de réflectivité ont une forme caractéristique reflétant l'épaisseur totale $D$ et les pics de Bragg indiquant directement la périodicité $d$ des bi-couches.

Quand on abaisse le champ magnétique externe vers une valeur proche d'un "champ de guide de neutrons" les aimantations des couches de Fe sont opposées les unes aux autres en raison des couplages d'échange [29]. Cela se traduit par l'apparition de pics de Bragg supplémentaires de valeur $1 / 2,3 / 2$ etc (voir Figure 4(b')) indiquant ainsi le doublement de la maille élementaire de la structure en multicouches (Eq. 3.4). Les calculs de la Figure 4(b') ne sont valides que pour un état mondomaine pour chaque couche magnétique.

Cependant, il serait difficile d'imaginer que dans ce dernier cas les couches magnétiques conservent un état monodomaine dans l'état rémanent [31]. Afin de minimiser l'énergie libre du matériau ferromagnétique, les couches magnétiques se fragmenteront en ensemble de domaines d'aimantations opposées mais dans la même direction que l'axe de facile aimantation. Dans ce cas, deux scenarios sont possibles : Grand et petits domaines.

Le premier scenario se met en place quand la taille du domaine est plus grande que $L_{s}^{\alpha}$. Cette conjecture peut âtre démontrée par l'absence de diffusion hors-spéculaire. Dans ce cas, il faut calculer les profils de réflectivité pour chaque type de domaines et les ajouter de façon incohérente.

Le second scenario apparaît quand on observe de la diffusion hors-spéculaire. Cela signifie que les inhomogénéites magnétiques latérales (domaines) sont plus petites que $L_{s}^{\alpha}$; alors l'onde n'est plus seulement réfléchie spéculairement mais aussi diffusée hors de la direction spéculaire (Figure 5). Ce cas doit âtre traité par le formalisme DWBA et sera discuté dans la section suivante.

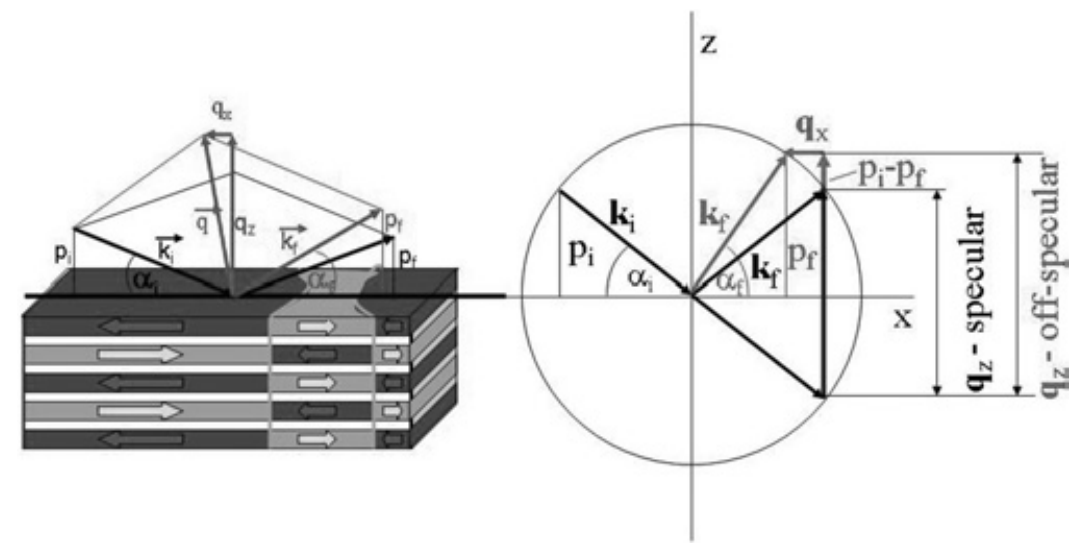

Figure 5. Multicouche magnétique avec domaines. Les vecteurs d'onde incident $\boldsymbol{k}_{i}$ et réfléchi de manière spéculaire $\boldsymbol{k}_{f}$ sont indiqués par des flèches noires. Les flèches rouges correspondent à la partie hors spéculaire. Sur la droite de la figure, le procéssus de diffusion dans l'espace réciproque. 


\subsection{Diffusion hors-spéculaire}

Les flucuations latérales de la SLD autour de sa valeur moyenne donne naissance à un vecteur de potentiel d'interaction perturbatif dans l'espace de spins.

$$
\widehat{V}_{p}(\mathbf{r})=\widehat{V}(\mathbf{r})-\widehat{V}_{0}(z)
$$

Les fonctions d'ondes $\psi\left(\mathbf{k}_{i} ; \boldsymbol{\rho}, z\right)$ et $\psi\left(\mathbf{k}_{f} ; \boldsymbol{\rho}, z\right)$ des ondes incidentes et diffusées pour un potentiel de la couche moyenne sur les coordonnées latérales $\rho$ ont été déterminées dans la section précédente. Utilisant ces fonctions d'ondes et la diffusion, l'amplitude de diffusion est

$$
\widehat{f}\left(\mathbf{k}^{f}, \mathbf{k}^{i}\right)=-\frac{m}{2 \pi \hbar^{2}} \int d \mathbf{r}\left\langle\psi^{f}\left(k^{f}, \boldsymbol{r}\right)\left|\widehat{V}_{s}(\mathbf{r})\right| \psi^{i}\left(k^{i}, \boldsymbol{r}\right)\right\rangle
$$

Le potentiel de perturbation est proportionnel à la densité de longueur de diffusion des fluctuations $\hat{F}(Q)$, et peut être écrit comme [25]

$$
\widehat{F}(Q)=b_{N} F_{N}(\mathbf{Q})+\left(\boldsymbol{\sigma} \boldsymbol{m}_{\perp}\right) b_{M} F_{M}(\mathbf{Q})
$$

où $b_{N}$ et $b_{M}$ sont les longueurs de diffusion nucléaire et magnétiques, $F_{N}(\mathbf{Q})$ est la facteur de structure nucléaire, $F_{M}(\mathbf{Q})$ est le facteur de forme magnétique, $\boldsymbol{\sigma}$ est le vecteur des matrices de Pauli, $\boldsymbol{m}_{\perp}$ est la composante de l'aimantation des couches qui est perpendiculaire au vecteur de transfert $Q$.

Pour une structure multicouche il faut prendre en compte la réfraction et la diffusion des ondes réfléchies par chaque interface. Cela est possible dans le cadre de la DWBA [25-32] et l'opérateur de diffusion pour chaque couche est de la forme :

$$
\widehat{F}_{f i}=\widehat{t}_{f} \widehat{F}^{t t} \widehat{t}_{i}+\widehat{t}_{f} \widehat{F}^{t r} \widehat{r}_{i}+\widehat{r}_{f} \widehat{F}^{r t} \widehat{t}_{i}+\widehat{r}_{f} \widehat{F}^{r r} \widehat{r}_{i}
$$

le point important est que la diffusion d'une couche magnétique s'accompagne d'une transition entre les états de spin $\{+,-\}$. A partir d'une définition générale [32] la section efficace de diffusion et la réflectivité s'écrivent :

$$
\frac{d \sigma}{d \Omega}=\operatorname{Tr}\left\{\widehat{\rho}^{i}\left(\widehat{F}_{f i}^{+}\right) \widehat{\rho}^{f} \widehat{F}_{f i}\right\} \quad R=\operatorname{Tr}\left\{\widehat{\rho}^{i}\left(\widehat{R}^{+}\right) \widehat{\rho}^{f} \widehat{R}\right\}
$$

où les matrices densité $\hat{\rho}^{i}$ et $\widehat{\rho}^{f}$ décrivent les proprietés du polariseur et de l'analyseur, respectivement.

$$
\hat{\rho}^{i}=\frac{1}{2}\left(1+\boldsymbol{\sigma} \boldsymbol{P}_{i}\right) \quad \hat{\rho}^{f}=\frac{1}{2}\left(1+\boldsymbol{\sigma} \boldsymbol{P}_{f}\right)
$$

La substitution de l'Eq. (3.9) dans l'Eq. (3.10) permet d'obtenir une équation générale des sections efficaces de diffusion [27], pour lesquelles les élements diagonaux sont [32]

$$
\begin{aligned}
\frac{d \sigma^{y y}}{d \Omega_{++}} & =\frac{d \sigma^{y y}}{d \Omega_{--}}=0 \\
\frac{d \sigma^{y y}}{d \Omega_{+-}} & =\left|F_{x}-i F_{z}\right|^{2} \\
\frac{d \sigma^{y y}}{d \Omega_{-+}} & =\left|F_{x}+i F_{z}\right|^{2} \\
\frac{d \sigma^{x x(z z)}}{d \Omega_{++}} & =\frac{d \sigma^{x x(z z)}}{d \Omega_{--}}=\left|F_{x(z)}\right|^{2} \\
\frac{d \sigma^{x x(z z)}}{d \Omega_{+-}} & =\frac{d \sigma^{x x(z z)}}{d \Omega_{-+}}=\left|F_{z(x)}\right|^{2}
\end{aligned}
$$

L'application de ces équations à des cas magnétiques et non-magnétiques permet une analyse de la réflectivité spéculaire et hors-spéculaire des systèmes en multicouches (voir section suivante). 


\section{EXEMPLES DE CALCULS NUMÉRIQUES}

Les différentes données expérimentales de la Figure 1 ont été collectées sur une grande gamme de vecteurs d'ondes incidents et diffusés, $y$ compris la zone de réflexion totale. Quelles sont les origines de la diffusion hors-spéculaire? On peut déterminer plusieurs groupes, voir Figure 6, qui sont emblématiques des principaux cas de "fluctuation de couches" existants.

Le premier cas est la rugosité d'interface (voir Figure 6(a)). La rugosité caractérise les irrégularités de surface liées aux procédés de fabrication. Ces irrégularités forment la texture de la surface. En général, la rugosité est identifiée par l'absence de structure latérale régulière et est traitée par ses proprietés de corrélations à l'échelle mésoscopique en utilisant des fonctions de distribution. Les nanoparticules insérées dans une matrice, voir Figure 6(b), changent la morphologie et peuvent initier une transformation de phase de la matrice hôte. Les fluctuations latérales d'un tel système seront caractérisées par un facteur de forme des particules et par la distribution de taille de ces particules. Ces fluctuations produisent une diffusion hors-spéculaire additionnelle à celle des interfaces.

\section{Conformal roughness}

a) Rough surfaces \& interfaces

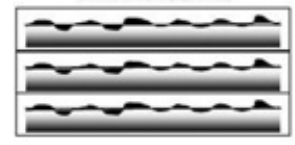

b) Embedded nanoparticles

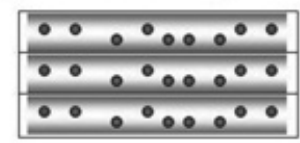

Non-conformal roughness
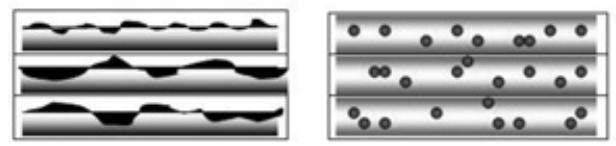

c) Magnetic domains

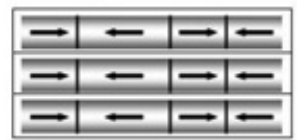

d) Stripe arrays \& Dots

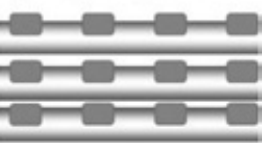

Figure 6. Différents types de fluctuations de densité (ou rugosité) dans les films et les multicouches. Les séquences dans la ligne du haut et celle du bas illustrent deux cas limites : rugosité parfaitement conformelle et non-conformelle.

La formation de domaines joue un rôle crucial dans le mécanisme de renversement d'aimantation. A l'exception du cas en champ magnétique saturant, la structure magnétique latérale des films et multicouches n'est pas stable et se décompose en une série de domaines latéraux (voir Figure 6(c)). La section efficace de diffusion magnétique est déterminée par le facteur de forme des domaines, le facteur de structure de la multicouche et une combinaison des amplitudes de réflectance et de transmittance pour chaque état de spin. La diffusion hors-spéculaire contient une information non seulement sur les domaines mais aussi sur la moyenne sur tous les domaines de l'aimantation moyenne [33].

Les structures périodiques à motifs ("patterned") comme les réseaux microniques ou sub-microniques de bandes, plots ou anneaux, et qui trouvent des applications dans les dispositifs de stockage optique ou magnétique, sont représentées sur la Figure 6(d). La diffusion hors-spéculaire de telles structures est déterminée par la facteur de forme et par la structure interne des objets latéraux ainsi que par le facteur de structure.

Un paramètre important dans la description des structures de surface ou d'interface est le paramètre de conformité. Il s'agit d'une mesure de la réplication du profil de rugosité d'une interface à l'autre dans une multicouche. Les schémas en haut et en bas de la figure 6 montrent les deux cas limites de systèmes conformels et non-conformels.

Dans ce qui suit nous discuterons différents effets de la diffusion hors-spéculaire. 
Partons du cas le plus simple, à savoir une multicouche ayant des interfaces idéales (voir Figure 7). La structure du film se compose de 12 bi-couches d'épaisseur $d$, avec une épaisseur totale $D$. En l'absence de rugosité seule la réflectivité spéculaire résultant par la structure transverse sera non nulle. Le résultat du calcul est représenté sur la partie droite de la Figure 7. Il s'agit d'une cartographie 2D avec pour axes $p_{i}$ et $p_{f}$, les composantes normales du vecteur d'onde des neutrons $\left(p_{i}=k \sin \alpha_{i}\right.$ et $p_{f}=k \sin \alpha_{f}$, où $\alpha_{i, f}$ sont les angles d'incidence et de réflexion, respectivement, comme indiqué sur la Figure 4). L'information principale contenue dans cette cartographie est l'oscillation de la réflectivité pilotées par la périodicité verticale de la SLD. L'intensité spéculaire le long de la ligne $p_{i}=p_{f}$ montre les oscillations liées a l'épaisseur totale $D, p_{i}+p_{f}=2 \pi / D$, ainsi que les pics de Bragg de $1^{e r}$ et $2^{e}$ ordre pour $p_{i}+p_{f}=2 \pi n / d$. La réflexion totale, très intense, est visible près de l'origine des axes.

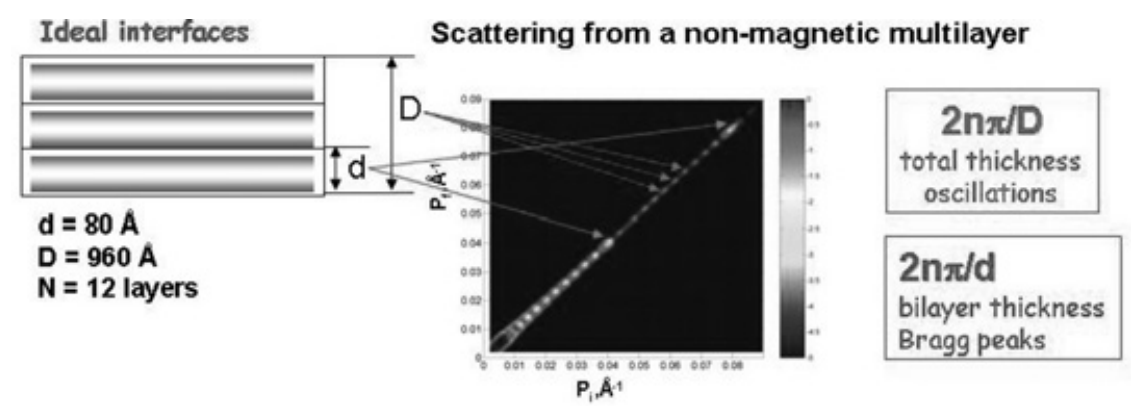

Figure 7. Intensité $2 D$ d'une multicouche avec des interfaces idéales. La forte intensité le long de la diagonale correspond à la contribution spéculaire, la région de réflection totale est proche de l'origine de la carte, suivie des oscillations dues à l'épaisseur totale $D$ et des deux pics de Bragg dus à l'épaisseur de la bicouche $d$.

$\mathrm{Si}$, sur une distance $L_{s}^{\alpha}$, les interfaces ne sont pas planes et que l'invariance translationnelle latérale est violée alors la rugosité induite par les fluctuations locales de la SLD autour de sa valeur moyenne donne lieu à une diffusion hors-spéculaire. La Figure 8 montre les calculs obtenus sur le même système que pour la Figure 7 mais avec des interfaces rugueuses conformelles. Les dimensions latérales et transverses de la rugosité sont $\xi=30 \mathrm{~nm}$ et $\sigma=0.5 \mathrm{~nm}$. La signature de la rugosité conformelle est que
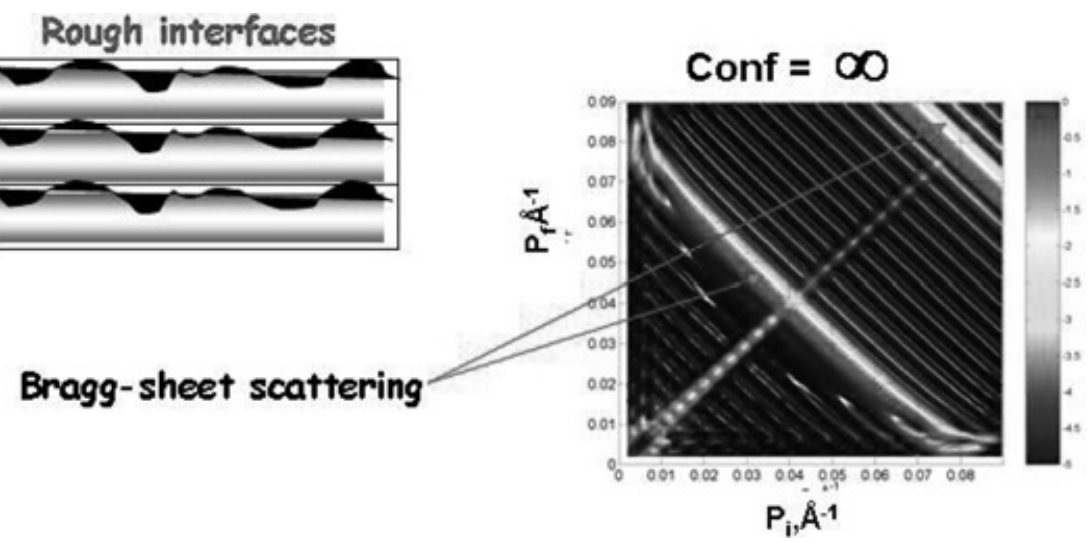

Figure 8. Carte 2D de l'intensité diffusée par une multicouche non magnétique présentant une rugosité de surface conformelle. La diffusion hors spéculaire donne lieu à des bandes croisant la ligne de réflectivité aux positions des maxima des oscillations d'épaisseur (faible intensité) et des pics de Bragg (indiqués par des flèches). 
la diffusion se compose de bandes d'intensité s'étirant perpendiculairement à la ligne spéculaire. Il y a deux types de diffusion hors-spéculaire. La première correspond à la diffusion le long de $\left(p_{i}+p_{f}\right)=$ $2 \pi N / D$, traversant les oscillations d'épaisseur de la ligne spéculaire. Elle apparaît en raison de la conformité entre les rugosités du substrat et de la surface. La deuxième est visible le long des lignes $\left(p_{i}+p_{f}\right)=2 \pi n / d$ traversant les pics de Bragg sur les bords de la ligne spéculaire (diffusion sur les plans de Bragg). Les lignes hors-spéculaire sont distordues en raison des effets de réfraction aux faibles valeurs de $p_{i}$ et/ou $p_{f}$ lorsqu' on s'approche de la zone de réflexion totale mais deviennent droites pour les valeurs élevées de $p_{i}$ et $p_{f}$.

Jusqu'à présent nous avons considéré les systèmes non-magnétiques mais, nous en avons déjà parlé, les films ou multicouches magnétiques peuvent présenter des structures en domaines ou des rugosités magnétiques d'interface, donnant lieu à une diffusion hors-spéculaire.

Considérons maintenant la diffusion par des multicouches magnétiques. Pour la simplicité, nous supposerons d'abord que les interfaces sont planes sur une échelle comparable à $L_{s}^{\alpha}$. Le système modélisé est représenté sur la Figure 9. Il consiste en 12 bi-couches de $\left.{ }^{57} \mathrm{Fe}(70 \AA) \mathrm{Cr}(10 \AA)\right]$, ayant une anisotropie planaire d'ordre 4, déposé sur un substrat de $\mathrm{Al}_{2} \mathrm{O}_{3}$. Il s'agit d'un exellent exemple de système présentant un effet GMR [2,3] lié à l'échange antiferromagnétique entre les moments magnétiques des couches de Fe voisines [33]. Le champ magnétique externe $\mathbf{H}$ est appliqué le long de la

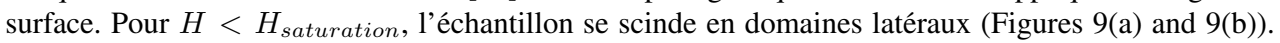
Pour plus de clarté, on suppose un état canté homogène avec un angle de "canting" $\pm \varphi$ entre couches adjacentes. Les domaines réduisent l'énergie dipolaire via la désaimantation de chaque couche $n$, et la valeur moyenne de l'aimantation est $\bar{M}_{n}^{\perp}=M \overline{\sin \varphi_{n}}$.

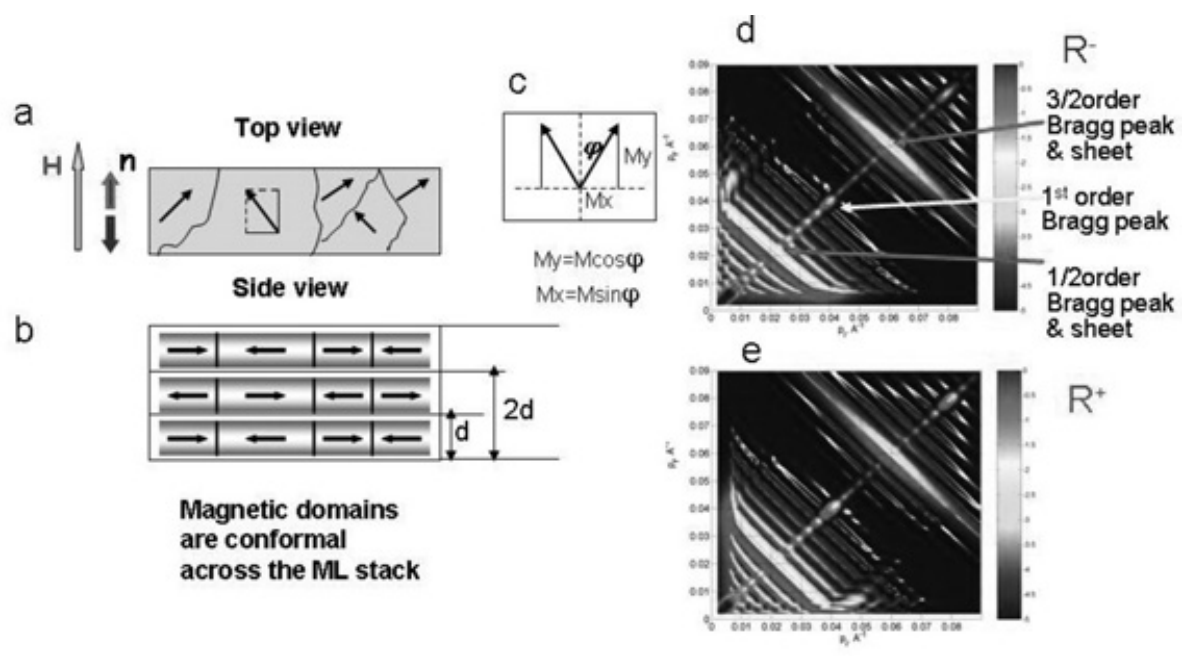

Figure 9. Diffusion par une multicouche magnétique ayant les interfaces idéales et présentant des domaines magnétiques. Les structures latérales et transverses sont indiquées en (a) (vue du dessus) et (b) (vue de coté). (c) Directions de l'aimantation dans deux types de domaines cantés d'un angle $\varphi$ par rapport à l'axe de quantification des neutrons. Cartes 2D de l'intensité réfléchie et diffusée pour des neutrons de spin antiparallèles (d) et parallèles (e) au champ magnétique extérieur. La très forte assymétrie de la diffusion hors-spéculaire provient de la nature spin-flip de la diffusion et des valeurs des longueurs de diffusion pour les deux états de spin.

Un exemple de simulation numérique de ce modèle pour des neutrons incidents dans l'état '-' et '+' est représenté sur les Figures 9(d) et 9(e) dans le cas où les domaines magnétiques (taille $280 \mathrm{~nm}$ ) sont conformes à travers l'épaisseur de l'empilement. La cartographie 2D montre plusieurs choses importantes. On voit très bien les plans de Bragg antiferromagnétiques le long de $\left(p_{ \pm}^{i}+p_{\mp}^{f}\right) d \approx \pi, 3 \pi$ 
ainsi qu'un ensemble de plans moins intenses et parallèles aux plans de Bragg. La distance entre eux est déterminée par l'épaisseur totale de la multicouche. L'autre élement important est que la diffusion hors-spéculaire est fortement asymétrique, révélant l'asymétrie de l'amplitude de diffusion "spin flip", $F_{z}$, par rapport aux variations de $\left(p_{ \pm}^{i}\right.$ et de $\left(p_{ \pm}^{f}\right.$ [32]. Les valeurs critiques pour lesquelles la diffusion hors-spéculaire est coupée sont reliées au fait que la SLD $N b$ est différente pour les deux états de spin des neutrons : $N b^{ \pm}=N b_{n} \pm N b_{m}$, où $\mathrm{N}$ est la densité du numéro atomique, $b_{n}$ et $b_{m}$ sont les longueurs de diffusion nucléaires et magnétiques, respectivement [34]. La diffusion hors-spéculaire "spin flip" est déterminée par la composante perpendiculaire du vecteur de l'aimantation.

L'autre effet observé sur les Figures 9(d) et 9(e) est la présence de plans de Bragg "anormaux". Ils s'étirent parallèlement au bord de réflectivité sur une distance $\left(p_{i}^{+}-p_{f}^{+}\right) D \approx \pi$ [35]. Ce type de diffusion apparaît quand l'onde diffusée est réfléchie par une interface et, en raison de l'origine "spin flip" de cette diffusion, elle est fortement asymétrique.

Maintenant nous allons combiner les deux cas discutés précédemment en prenant une multicouche ayant des interfaces rugueuses et des domaines magnétiques. le système est présenté sur la Figure 10. La cartographie $2 D$ de l'intensité diffusée pour les neutrons "down" est une superposition du signal "spin flip" et "non spin flip" émanant des deux sources de rugosité interfaciale (non magnétique) et des domaines magnétiques colonnaires comme cela vient d'être discuté. Cette exemple montre que la diffusion hors-spéculaire discrimine la rugosité au sens où les différentes parties du résultat sont déterminées par des processus différents et peuvent être analysés dans cette expérience [33-35].

a

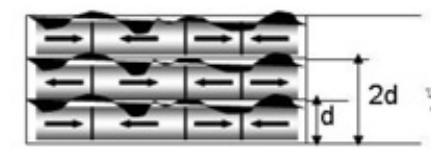

b

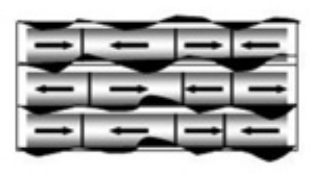

C

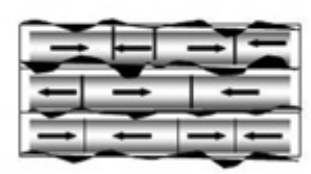

(1)
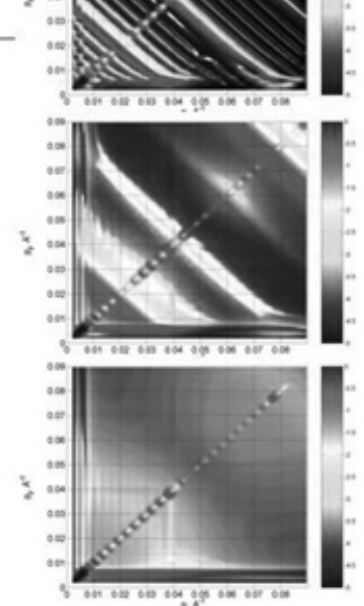

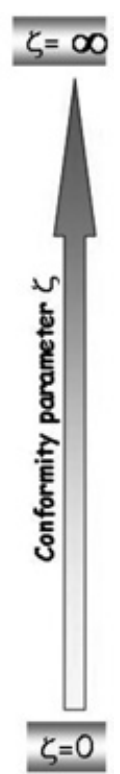

Figure 10. Diffusion par une multicouche magnétique ayant une interface rugueuse et présentant des domaines magnétiques. Sur la gauche de la figure, vue dans l'espace réel des multicouches de même structure mais de conformité différente (rugosité de l'interface et domaines magnétiques). (a) système de conformité idéale ; (b) multicouche avec un degré de conformité partiel ; (c) système non conformel présentant des fluctuations magnétiques et non magnétiques. Sur la droite de la figure, les cartes d'intensité 2D correspondantes, qui sont calculées avec des paramètres de conformité $\zeta$ différents.

L'apparition des signes caractéristiques de la diffusion hors-spéculaire dépend non seulement des fluctuations latérales de la SLD mais aussi des corrélations verticales (paramètre de conformité). Pour 
l'instant nous n'avons considéeré que la rugosité conformelle, mais si cela n'est plus le cas, i.e. si les différentes interfaces n'ont plus qu'une corrélation partielle sur une distance typique $\Lambda_{\perp}$, alors nous devons introduire une fonction de correlation inter-plans [36] :

$$
\frac{d \sigma}{d \Omega}=e^{-\left|z_{m}-z_{n}\right| / \Lambda_{\perp}} \sum_{l, l^{\prime}=1} \operatorname{Tr}\left(\rho^{i} \widetilde{F}_{l^{\prime}}^{+} \rho^{f} \widetilde{F}_{l^{\prime}}\right)
$$

où $\widetilde{F}_{l^{\prime}}$ est l'opérateur de diffusion, $\rho^{i, f}$ sont les matrices de densité et $z$ est la coordonnée transverse. Figure 10(b) et 10(c) montrent les calculs de la diffusion hors-spéculaire pour le même système que sur la Figure 10(a) mais en supposant différents degrés de conformité. Sur la Figure 10(b), les flucuations de SLD (magnétique et/ou non-magnétique) sont partiellement corrélées d'une couche à l'autre, i.e. les phases des ondes diffusées sont partiellement cohérentes. Dans ce cas, en plus de la diffusion liée aux plans, une intensité additionnelle issue de la rugosité non-corrélée apparaît et s'étirent le long $p^{i, f}=p^{c}$. Il s'agit de la diffusion de Yoneda.

Le dernier cas (voir Figure 10(c)) correspond à un système ayant une rugosité non-corrélée quand les interfaces diffusent indépendamment. La diffusion sur les plans de Bragg disparaît, la diffusion horsspéculaire provenant des interfaces individuelles se superpose et la diffusion de Yoneda devient plus intense. Cette diffusion est assez complexe car la partie asymétrique vient de la diffusion "spin flip" de domaines magnétiques non-corrélés et que la partie symétrique est déterminée par la rugosité interfaciale non-magnétique (non-corrélée également).

Dans les exemples numériques décrits nous avons montré les principaux phénomènes apparaissant sur différents motifs de diffusion hors-spéculaire que ce soit par des systèmes magnétiques ou nonmagnétiques. Dans la prochaine section, nous discuterons les résultats expérimentaux.

\section{RÉSULTATS EXPÉRIMENTAUX : EXEMPLES}

\subsection{Multicouches de copolymères autoassemblés}

En raison des implications tant fondamentales qu'appliquées liées à la formation des surfaces et interfaces de polymères, les structures de multicouches di-blocks ont été intensivement étudiées par un grand nombre de techniques telles la microscopie à force atomique (AFM), la microscopie à transmission d'électrons (TEM), la réflectivité spéculaire des neutrons ou de rayons-X. Malheureusement, aucune d'entre elles ne peut donner de détails quantitatifs à la fois sur les structures transverses et latérales de surface et en profondeur. Cette information peut être obtenue par la diffusion hors-spéculaire des neutrons dans la mesure où les différentes contributions sont identifiables et modélisées. [26,36].

Les polymères di-blocks symétriques consistant en deux chaînes de polymères chimiquement différentes et liées entre elles sont en fait des micro-phases séparées en domaines en raison de l'immiscibilité des deux polymères. Le mélange de ces polymères avec un solvant est déposé par centrifugation sur un substrat plat. Ensuite, durant le recuit au-dessus de la température de transition vitreuse la séparation en micro-phases conduit à un ordre lamellaire sur le substrat.

Une couche incomplète présentant une interface rugueuse est caractéristique de la plupart des films de copolymères diblocks symétriques après le recuit. Une surface consistant en îlots, trous ou structures labyrinthiques et la rugosité interfaciale des couches de profondeur génère une diffusion hors-spéculaire. L'utilisation d'un modèle basé sur la théorie dynamique de la diffusion pour l'analyse des cartographies $2 D$, nous permet d'identifier et de modéliser les différentes sources de diffusion hors-spéculaire et d'obtenir une description compléte de la topologie de surface et de la morphologie de la rugosité d'interface.

Le système considéré est un film mince de copolymères diblock symétrique de polystyrène deutérié, d-PS, et de polybuthylméthacrylate, PBMA, dénoté P(d-S-b-BMA), ayant un poids moleculaire $M w=$ $248300 \mathrm{~g} / \mathrm{mol}$, une polydispersité $M w / M n=1.03$ et un ratio de block symétrique $(f=0.5)$. 


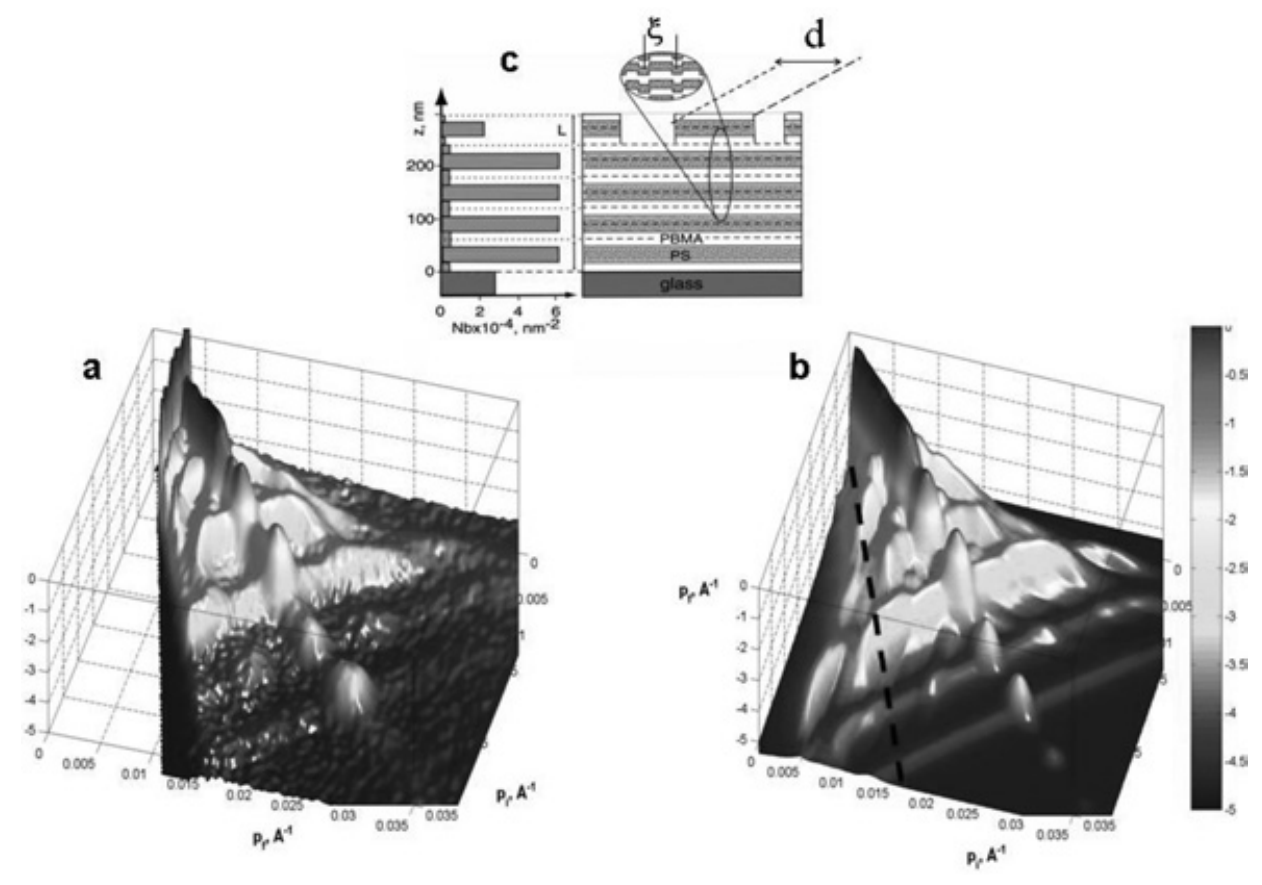

Figure 11. Cartes d'intensité 3D mesurées expérimentalement (a) et modélisées de la réflection spéculaire et de la diffusion hors-spéculaire d'une multicouche recuite de P(d-S-b-BMA). (c) Profil de densité de longueur de diffusion neutron de la structure transverse de la multicouche en fonction de la distance au substrat déduite de l'affinement d'un modèle à partir des données expérimentales ; (d) structure latérale de la multicouche présentant des fluctuations de rugosité d'interface de distance latérale moyenne $\xi$, ainsi qu'une structure en ilots de dimension latérale moyenne $\mathrm{d}$.

L'échantillon a été préparé par la méthode de dépôt par centrifugation d'une solution de toluène de $\mathrm{P}(\mathrm{d}-\mathrm{S}-\mathrm{b}-\mathrm{BMA})$ sur un substrat de verre puis recuit à $151^{\circ} \mathrm{C}$ pendant 94 heures.

Les intensités réfléchies et diffusées sont représentées sur la Figure 11(a) en fonction de $p_{i}$ et $p_{f}$. La réflexion spéculaire se trouve sur la ligne $p_{i}=p_{f}$ et le transfert de moment perpendiculaire est $\left(p_{i}+p_{f}\right)=Q_{z}$. La diffusion hors-spéculaire représentée en fonction de $\left(p_{i}-p_{f}\right)$ s'étale de chaque coté de la ligne spéculaire. La Figure 11(b) représente une simulation à partir du modèle de la Figure 11(c). Les résultats sont discutés dans la section suivante.

Un ajustement des paramètres sur la courbe de réflectivité révèle que le film de polymères consiste en 4 couches complètes de P(d-S-b-BMA) et une couche de surface avec des trous. La SLD réduite de la couche de surface reflète la présence de trous sur $\mathbf{1 0 \%}$ de la surface du film. La séquence de pics de Bragg est définie par l'épaisseur $L$ de la multicouche PS-PBMA et la distribution d'intensité de ces pics est déterminée par les amplitudes de SLD au dessus du substrat. La valeur de $L$ varie légèrement le long de l'épaisseur du film. Les interfaces sont modélisées par des fonction-erreur pour lesquelles la largeur représente la rugosité.

La structure des SLD obtenue entre dans l'équation d'onde afin d'établir la solution à l'ordre zéro du processus de diffusion. Ici, nous introduisons non pas un potentiel d'interfaces parfaites [23] mais un potentiel moyenné latéralement.

Pour distinguer entre les effets dus à la rugosité et aux îlots, il faut identifier la signature de ces deux effets sur la Figure 11. 
Le modèle incluant une rugosité d'interfaces (conformelle et non conformelle) conduit au cas représenté sur la Figure 12(a), 12(a'). La longueur de corrélation verticale exprimée par ce paramètre de conformité, qui peut s'exprimer en nombre d'interfaces, donne un ratio entre les deux mécanismes de diffusion. Le meilleur accord est trouvé avec un paramètre de conformité de 6 .

Le fait saillant de la rugosité conformelle est la diffusion sur les plans de Bragg à travers le pic de Bragg " $\mathrm{n}$ " sur la courbe de réflectivité et s'étendant le long de $\left(p_{i}+p_{f}\right)_{n B r a g g}$. Des déviations à ce comportement apparaissent à chaque fois que les plans de Bragg recoupent la diffusion émanant de la diffusion dynamique, comme la diffusion de Yoneda ou la diffusions anormale de plans de Bragg. La diffusion de Yoneda et de super-Yoneda est simplement de la diffusion de rugosité non conformelle, audel à de la ligne $p_{i, f}=p_{c}$ et des pics de Bragg $\left(p_{i}\right.$ ou $\left.p_{f}=\left(p_{i, f}\right)_{n B r a g g}\right)$, respectivement. L'avantage de tracer $\left(p_{i}+p_{f}\right)=f\left(p_{i}-p_{f}\right)$ est que la fonction $\left(p_{i}-p_{f}\right)$ est une fonction linéaire de $\alpha_{i}$ et de $\alpha_{f}$ alors que le transfert de moment latéral $Q_{x}$ est une fonction non-lineaire de ces angles. Donc, la diffusion de Yoneda apparaît ainsi comme une série de lignes droites. En revanche, cette diffusion est affectée par le croisement avec la diffusion, normale et anormale, des plans de Bragg. Cette dernière est parallèle à la courbe de réflectivité le long de $\left(p_{i}-p_{f}\right)=2 n p / L$. Des pics apparaissent si un des plans de Bragg intersectant est supprimé tandis que l'autre est augmenté par le facteur de structure. Sinon, l'interférence entre diffusion "normale" et "anormale" est destructrice. Mis à part ces détails, l'intensité globale le long des plans de Bragg dépend essentiellement de la longueur de corrélation latérale $\xi$ et de l'exposant fractionnaire $\nu$ de la corrélation de hauteur de la rugosité [23].

a
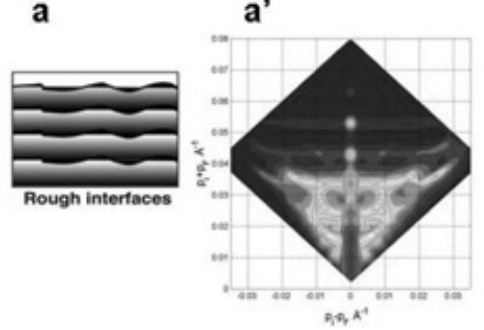

b

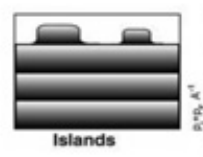

Islands

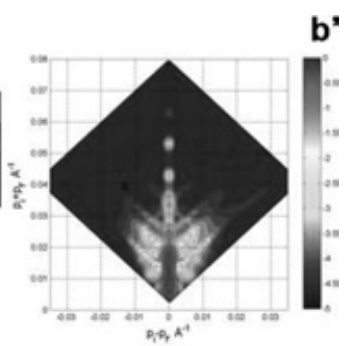

Figure 12. Le modèle et les résultats des calculs pour les contributions repésentées sur la Figure 11 (b). (a) et (a') diffusion hors spéculaire provenant de la rugosité d'interface entre PS et PBMA ; (b) et (b') diffusion hors spéculaire provenant des trous (ou îles) à la surface de la multicouche.

La diffusion hors-spéculaire générée par les trous (voir dessin de la Figure 12(b)) peut aussi bien être identifiée (voir Figure 12(b')). Elle est déterminée par les diffusions Yoneda et super-Yoneda. Les paramètres du potentiel perturbatif sont la profondeur des trous et le diamètre moyen des trous. La profondeur des trous est de $L=610 \AA$ et coïncide avec l'épaisseur d'une couche de (PBMA-PS-PSPBMA). En supposant une distribution aléatoire des trous, leur diamètre moyen est de $d=9000 \AA$ A La distribution de taille est décrite par une lorentzienne avec un exposant -1.5 .

Ayant identifié les principaux effets de la diffusion par la rugosité d'interface et la structure de surface, un ajustement des paramètres peut être effectué à partir de la Figure 11. Les paramètres du potentiel de perturbation utilisés dans le modèle DWBA [23,26,31] sont une hauteur moyenne $\sigma=$ $30 \AA$ centrée sur les interfaces avec une distance latérale moyenne de $\xi=1500 \AA$ et $\nu=1.5$ [35]. Le déclin croissant des plans de Bragg d'ordre supérieur est principalement déterminé par le paramètre de conformité et par la rugosité $\sigma=30 \AA$ A. Les interfaces du PBMA-PS oscillent donc avec une amplitude moyenne de $30 \AA$ sur une surface moyenne de l'ordre de $1500 \AA$ de diamètre. Cette oscillation est presque entièrement conformelle à travers la multicouche.

Cet exemple démontre que la diffusion hors-spéculaire dépend de la rugosité et que différentes sources de perturbation donnent lieu à différentes signatures dans l'intensité diffusée. 
5.2 Diffusion hors-spéculaire des neutrons no-polarisés, résolue en spin par des multicouches magnétiques

Dans le régime de la diffusion de Yoneda et de la diffusion par les plans de Bragg liés à la superstructure, la diffusion par des multicouches de neutrons non-polarisés dépend de l'état de spin. Les anomalies dans la diffusion hors-spéculaire, issues des fluctuations magnétiques dans les multicouches, sont déterminées par des processus "spin flip" sélectifs.

Dans les multicouches magnétiques, le rapport de force entre l'anisotropie magnéto-cristalline, les couplages d'échange entre couches et le champ magnétique externe conduit à ce que les aimantations des couches de Fe successives, dans chaque domaine colonnaire [33], présentent un certain angle de couplage avec ses voisines, comme le montre la Figure 13. Donc, même la diffusion des neutrons non polarisés peut âtre utilisée efficacement pour déterminer la structure magnétique [37].

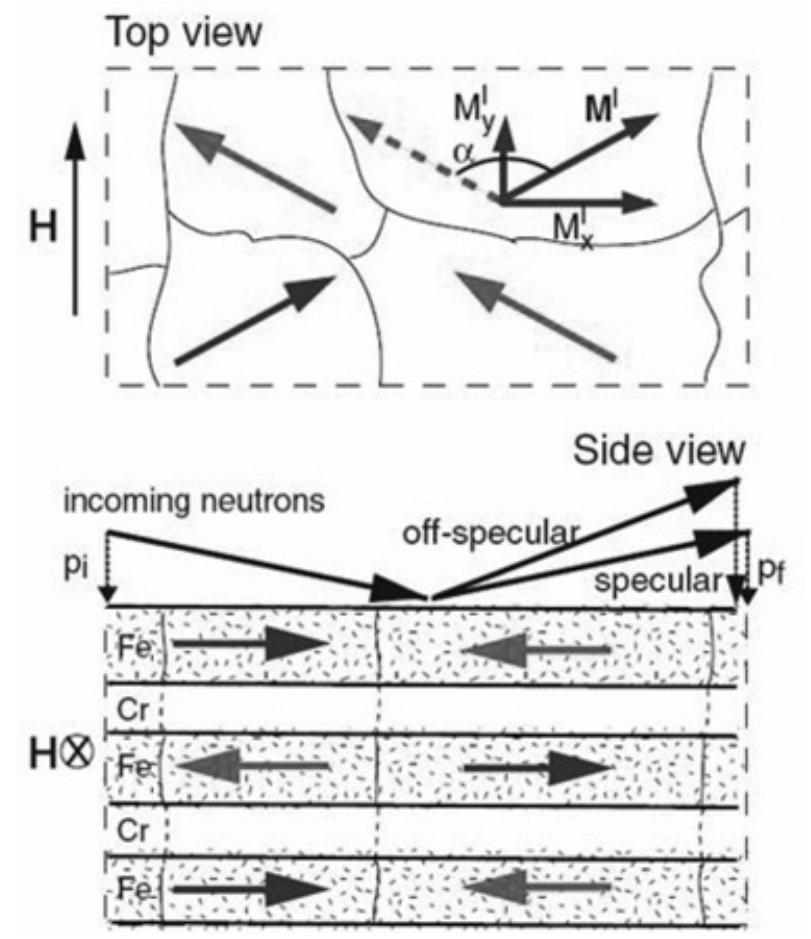

Figure 13. (Haut) distribution de l'aimantation $M^{l}$ dans les différents domaines d'une multicouche $\mathrm{Fe} / \mathrm{Cr}$ dans un champ magnétique extérieur $\boldsymbol{H}$ appliqué dans le plan ; la composition de l'échantillon est présentée schématiquement ; les angles de couplage $\alpha$ relient les moments magnétiques d'une couche (Ĺ l'intérieur d'un domaine) au moment magnétique de la couche adjacente. (Bas) Géométrie de la diffusion et composantes des moments magnétiques perpendiculaires au champ magnétique externe.

Des expériences de réflectivité ont été menées sur le spectrometre EVA (ILL, Grenoble) à la longueur d'ondes $5.4 \AA$ et un champ magnétique externe de 500 Gauss sur un échantillon multicouche, ([Cr $\left.\left.(9 \AA) /{ }^{57} \mathrm{Fe}(68 \AA)\right]-x 12 / \mathrm{Cr}(68 \AA)\right)$, déposé par épitaxie par jets moleculaire sur un substrat de saphir. La géometrie de diffusion est schématisée sur la Figure 13: L'aimantation des couches est décomposée en domaines (Figure 13, vue de dessus) en présence d'un couplage antiferromagnétique entre couches (Figure 13, vue de coté). L'angle de couplage $\alpha$ (voir Figure 13) entre les aimantations de couches de Fe 
successives le long de l'épaisseur est piloté par le champ magnétique externe. La Figure 14(a) montre une cartographie $2 D$ de la diffusion en fonction de $p_{i}$ et $p_{f}$. L'intensité de la réflexion spéculaire le long de $p_{i}=p_{f}$ montre les oscillations typiques dues à l'épaisseur totale mais aussi le pic de Bragg de premier ordre $\left(p_{i}=p_{f}=0.041 \AA^{-1}\right)$ correspondant à une épaisseur de bicouche de $77 \AA$. La diffusion hors-spéculaire provient de la structure en domaines. Il apparaît une diffusion "spin flip" des plans de Bragg à travers les pics de Bragg d'ordre $1 / 2$ et $3 / 2\left(\left(p_{i}=p_{f}=0.0205 \AA^{-1}\right.\right.$ et $p_{i}=p_{f}=0.0615 \AA^{-1}$, respectivement) sur la courbe de réflectivité ainsi qu'une diffusion Yoneda, "spin flip", parallèle à $p_{i}$ et $p_{f}$ (Figure 14(a)). Une preuve du caractère "spin flip" est apportée par l'analyse de spin des neutrons polarisés diffusés par la multicouche [33] mais, ici, cela est directement visible à la lumière de la chute d'intensité créée par les angles critiques. Les deux angles critiques sont déterminés par les densités de longueur de diffusion $S L D_{n} \pm S L D_{m p}$ pour les deux états de spins. $S L D_{n}$ est la contribution nucléaire de ${ }^{57} \mathrm{Fe}$ et $S L D_{m p}$ est la contribution magnétique provenant de $M_{y}^{l}$, la composante parallèle au champ magnétique (Figure 13). Donc, un angle critique le long de $p_{i}=0.009 \AA^{-1}$ et de $p_{f}=0.009 \AA^{-1}$ est observé en raison de l'utilisation de neutrons non-polarisés. Les pics caractéristiques (marque C sur la Figure 14) où la diffusion par les plans de Bragg rencontre la diffusion de Yoneda voient leur intensité diminuer pour un état de spin. Toute intensité sous la marque "C" provient de l'autre état de spin. Pour cet échantillon l'autre angle critique est imaginaire car la différence $S L D_{n}-S L D_{m p}$ est négative, donc la diffusion hors-spéculaire s'étend sous l'horizon le long de $p_{i}=0$ et $p_{f}=0$ et est visible sous la forme de bandes modulées parallèles à la diffusion modifiée de Yoneda (marques $A$ et $B$ sur la Figure 14(c)). Les positions de ces bandes de Yoneda dépendent de la valeur de l'angle critique. Ces effets ont été pris en compte pour réaliser la simulation représentée sur la Figure 14(b). Un autre paramètre important est donné par le potentiel du substrat qui conduit à un autre "cut off" de l'intensité. Pour un champ de $500 \mathrm{G}$, cette valeur coïncide avec le premier angle critique "C". Un zoom sur cette region est visible sur la Figure 14(c).
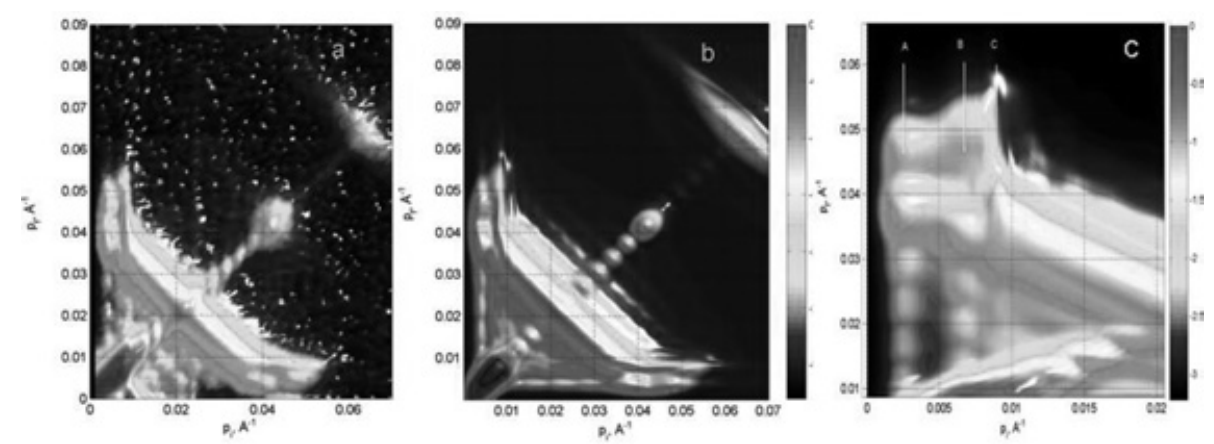

Figure 14. (a) Carte expérimentale à 2 dimensions d'une multicouche $\mathrm{Fe} / \mathrm{Cr}$ en fonction de $\boldsymbol{p}_{i}$ et de $\boldsymbol{p}_{f}$, les composantes perpendiculaires des vecteurs d'ondes incidents et diffusés. La forte intensité le long de la ligne $\boldsymbol{p}_{i}=\boldsymbol{p}_{f}$ correspond à la réflection spéculaire. La position du pic de Bragg du premier ordre correspond à l'épaisseur de la bicouche $\mathrm{Fe} / \mathrm{Cr}$ et ne présente pas de diffusion hors spéculaire. La diffusion magnétique hors spéculaire spin-flip s'étend des positions des pics de Bragg d'ordre $1 / 2$ et $3 / 2$ perpendiculaires à la ligne spéculaire. (b) Simulation à partir d'un modèle de la carte 2D d'intensité d'une multicouche $\mathrm{Fe} / \mathrm{Cr}$ observée sur la Figure 2(a). (c) Vue étendue de la région des données expérimentales où la diffusion par les plans de Bragg rencontre la diffusion de Yoneda. A et $\mathrm{B}$ montrent les positions de la diffusion Yoneda liées aux oscillations d'épaisseur totale, $\mathrm{C}$ est la position du premier angle critique qui correspond à la position de la coupure due au potentiel du substrat.

Avec le modèle DWBA [32], il est donc possible de connaître précisement l'aimantation des multicouches. En particulier, les densités de longueur de diffusion de ${ }^{57} \mathrm{Fe}\left(S L D_{n}\right.$ et $\left.S L D_{m p}\right)$ à partir des données de neutrons non-polarisés. La connaissance des $S L D_{m p}$ est critique pour déterminer l'angle de couplage $\alpha$ et le relier aux proprietés GMR du matériau [34]. 
En résumé, nous avons demontré qu'une information détaillée sur la morphologie des matériaux nanostructurés peut être obtenue en utilisant la diffusion spéculaire et hors-spéculaire des neutrons. Les détails de la structure interne des multicouches sont révélés par ces expériences de surface innovantes. Le potentiel de ce type de structures dépend de la capacité de contrôler non seulement la distribution des nano-objets mais aussi leurs morphologies et les caractéristiques de la structure "hôte".

Cette approche sera appliquée à un large eventail de systèmes technologiquement importants et peut servir de point de départ au développement de nouveaux matériaux.

\section{REMERCIEMENTS}

Je souhaite remercier Hans Lauter et Boris Toperverg pour leur longue et fructueuse collaboration dans ce travail et Victor Aksenov et Wienfried Petry pour leur soutien depuis de nombreuses années. Je suis redevable de Vladimir Ustinov, Lazar Romashev et Michail Milyaev pour leur collaboration sur les multicouches magnétiques. Je remercie mes collègues Alexander Petrenko et Alexei Vorobiev pour leur aide durant les expériences, ainsi que Oleg Nikonov pour son apport lors des premiers travaux sur la diffusion hors-spéculaire. Je remercie Eric Ressouche pour ses encouragements lors de la rédaction de cet article et sa patience pour la préparation de la version française. Ce travail a été soutenu par la BMBF (Project 03DU03MU).

\section{Références}

[1] Ankner, J., Felcher, G., JMMM 200 (1999) 741.

[2] Baibich, M.N., Broto, J.M., Fert, A., Nguen Van dau, F., Petroff, F., Etienne, P., Creuzet, G., Friederich, A. and Chazelas, J. Phys. Rev. Lett 61, 2472 (1988).

[3] Binasch, G., Grünberg, P., Saurenbach, F. and Zinn, W., Phys. Rev. B 39, 4828 (1989).

[4] Ambrose, T., Kai Liu, and Chien, C. L., J. Appl. Phys. 85, 6124 (1999).

[5] Strijkers, G.J., Zhou, S.M., Yang, F.Y. and Chien, C.L., Phys. Rev. B 62, 13896 (2000).

[6] Theis-Bröhl, K., Toperverg, B.P., Leiner, V., Westphalen, A., Zabel, H., et al., Phys. Rev. B 71,020404(R) (2005).

[7] Valden, M., Lai, X., Goodman, D.W., Science 281, 1647 (1998).

[8] Hansen, P.L., et al, Science 295, 2053 (2002).

[9] Semiconductor Quantum Dots, Zunger, A., Ed. Mater. Res. Soc. Bull 23 (no2) (1998).

[10] Toperverg, B.P., Felcher, G.P., Metlushko, V.V., Leiner, V., Siebricht, R., Nikonov, O., Physica B 283, 149 (2000).

[11] Kelberg, E.A., Grigoriev, S.V., Okorokov, A.I., et al., Physica B 335, 123 (2003); Grigoriev, S., Grigorieva, N., Vorobiev, A., (http://club.ill.fr/cv/servlet/ReportFind), ILL Exp Report 7-09-114 (2004).

[12] Sackmann, E., Science 271, 43 (1996).

[13] Wagner, M. and Tamm, I., Biophys. J 79, 1400 (2000).

[14] Gutberlet, T., Steitz, R., Fragneto, G., Klosgen, B., J. Phys.: Condens. Matter 16, S2469 (2004).

[15] Nelson, D.L. and Cox, M.M., Lehninger Principles of Biochemistry, 4th ed. (W.H. Freeman and Company, San Francisco, 2004), Chap.II.

[16] Su, L.Y., Hawkridge, F.M. and Rohten, M.C., Chem. Biodiversity, 1, 1281 (2004).

[17] Beugin, S., Edwards, K., Karlsson M. Ollivon, G., Lesieur, S., Biophys J 74, 3198 (1998).

[18] McGillivray, D.J., Thomas, R.K., Rennie, A.R., Penfold, J., Sivia, D.S., Langmuir 19, 7719 (2003).

[19] Mandel, L. and Wolf, E., Optical coherence and Quantum optics (Cambridge Univ. Press, Cambridge 1995). 
[20] Schiff, L.I., Quantum Mechanics, McGraw_Hill, New York, 1968.

[21] Vineyard, G.H., Phys. Rev. B 26, 4146 (1982).

[22] Mohanty, U. and Rice, S.A., J. Chem. Phys. 79, 2482 (1983).

[23] Sinha, S.K., Sirota, E.B., Garoff, S., Stanley, E., Phys. Rev. B 38, 2297 (1988).

[24] Pynn, R., Phys. Rev. B 45, 602 (1992).

[25] Toperverg, B.P., Physica B 297, 160 (2001).

[26] Toperverg, B.P., Lauter-Pasyuk, V., Lauter, H., Nikonov, O., Ausserre, D., Gallot, Y., Physica B 283, 60 (2000).

[27] Toperverg, B.P., Rühm, A., Donner, W., Dosch, H., Physica B 267-268, 198 (1999) ; Rühm, A., Toperverg, B.P., Dosch, H., Phys. Rev. B 60, 16073 (1999).

[28] Parratt, L.G., Phys. Rev. 95, 359 (1954).

[29] Grünberg, P., Schreiber, R., Pang, Y., Brodsky, M., Sowers, H., Phys. Rev. Lett. 57, 2442 (1986); Baibich, M.N., Broto, J.M., Fert, A., Nguen Van Dau, F., Petroff, F., Etienne, P., Creuset, G., Friederich, A., Chazelas, J., Phys. Rev. Lett. 61, 2472 (1988) ; Binach, G., Grünberg, P., Saurenbach, F., Zinn, W., Phys. Rev. B 39, 4828 (1989).

[30] Kiessig, H., Ann. Phys. Leipzig 10, 769 (1931).

[31] te Velthuis, S.G.E., Jiang, J.S., Bader, S.D., Felcher, G.P., Phys. Rev. Lett, 89, 127203 (2002).

[32] Toperverg, B.P., Nikonov, O., Lauter-Pasyuk, V., Lauter, H., Physica B 297, 169 (2001).

[33] Lauter-Pasyuk, V.V., Lauter, H.J., Toperverg, B.P., Romashev, L., Ustinov, V., Phys. Rev. Lett. 89, 167203 (2002).

[34] Lauter-Pasyuk, V.V., Lauter, H.J., Toperverg, B.P., Nikonov, O., Kravtsov, E., Romashev, L., Ustinov, V., J. Magn. Magn. Mater. 226-230, 1694 (2001).

[35] Lauter-Pasyuk, V.V., Lauter, H.J., Toperverg, B.P., Petrenko, A., Schubert, D., Schreiber, J., Burcin, M., Aksenov, V., Appl. Phys. A 74 [Suppl.], S528 (2002).

[36] Ming, Z.H., Krol, A., Soo, Y.L., Kao, Y.H., Park, J.S., Wang, K.L., Phys. Rev. B 47, 16373 (1993).

[37] Lauter, H.J., Lauter-Pasyuk, V.V., Toperverg, B.P., Romashev, L., Ustinov, V., Kravtsov, E., Vorobiev, A., Nikonov, O., J. Major, Appl. Phys. A 74 [Suppl.], S1557 (2002). 